\title{
Left ventricular diastolic dysfunction in idiopathic pulmonary fibrosis: a tissue Doppler echocardiographic study
}

\author{
C.E. Papadopoulos*, G. Pitsiou\# , T.D. Karamitsos*, H.I. Karvounis*, \\ T. Kontakiotis", G. Giannakoulas*, G.K. Efthimiadis*, P. Argyropoulou\#, \\ G.E. Parharidis* and D. Bouros
}

ABSTRACT: It was hypothesised that, apart from right ventricular (RV) dysfunction, patients with idiopathic pulmonary fibrosis (IPF) also exhibit left ventricular (LV) impairment, which may affect disease progression and prognosis. The aim of the present study was to evaluate LV performance in a cohort of IPF patients using conventional and tissue Doppler echocardiography.

IPF patients exhibiting mild-to-moderate pulmonary arterial hypertension (mean age $65 \pm 9 \mathrm{yrs}$; $\mathrm{n}=22$ ) and healthy individuals (mean age $61 \pm 6 \mathrm{yrs} ; \mathrm{n}=22$ ) were studied. Conventional and tissue Doppler echocardiography were used for the evaluation of RV and LV systolic and diastolic function. In addition to the expected impairment in RV function, all patients showed a characteristic reversal of LV diastolic filling to late diastole compared with controls (early diastolic peak filling velocity (E)/late diastolic peak filling velocity $0.7 \pm 0.2$ versus $1.5 \pm 0.1$, respectively). Patients with IPF also exhibited lower peak myocardial velocities in early diastole $(\mathrm{Em}$; $5.7 \pm 1.1$ versus $10.3 \pm 1.6 \mathrm{~cm} \cdot \mathrm{s}^{-1}$, respectively), higher in late diastole $\left(A \mathrm{~m} ; 8.9 \pm 1.3\right.$ versus $5.5 \pm 0.8 \mathrm{~cm} \cdot \mathrm{s}^{-1}$, respectively), lower $\mathrm{Em} / \mathrm{Am}$ ratio (0.6 \pm 0.1 versus $1.9 \pm 0.5$, respectively) and higher $\mathrm{E} / \mathrm{Em}$ ratio (10.8 \pm 3 versus $6 \pm 0.6$, respectively), all indicative of LV diastolic dysfunction. Moreover, LV propagation velocity was significantly lower in IPF patients $\left(46 \pm 13\right.$ versus $83 \pm 21 \mathrm{~cm} \cdot \mathrm{s}^{-1}$, respectively).

Physicians should be aware that patients with idiopathic pulmonary fibrosis exhibit early impairment of left ventricular diastolic function.

KEYWORDS: Diastole, Doppler echocardiography, idiopathic pulmonary fibrosis, left ventricular dysfunction

diopathic pulmonary fibrosis (IPF) is a progressive fibrosing inflammatory lung disease leading progressively to pulmonary hypertension $(\mathrm{PH})$, with increased morbidity and mortality and a mean survival duration after diagnosis ranging 3-5 yrs [1-3].

Right ventricular (RV) dysfunction has been well described in IPF patients, and in several conditions affecting pulmonary circulation, such as primary $\mathrm{PH}$, chronic obstructive pulmonary disease, chronic thromboembolic $\mathrm{PH}$, systemic sclerosis, systemic lupus erythematosus and cystic fibrosis [4-10]. Moreover, left ventricular (LV) diastolic dysfunction has been reported in some of these conditions [5-7, 11, 12], mainly as a consequence of RV pressure overload. Nevertheless, the prevalence of LV dysfunction in patients with IPF and mild-to-moderate $\mathrm{PH}$ is not known. Importantly, LV dysfunction, if present, could be an additional factor that may further impair exercise performance and affect prognosis early in the course of the disease.

New noninvasive echocardiographic techniques that are relatively load-independent, such as tissue Doppler imaging (TDI) and colour M-mode, have made echocardiography the clinical standard for the assessment of LV diastolic function [13, 14]. Regional myocardial velocities and time intervals during systole and diastole can be accurately and reproducibly measured by TDI, permitting the
AFFILIATIONS

*1st Cardiology Dept, AHEPA University Hospital,

${ }^{\#}$ Respiratory Failure Unit, Aristotle University of Thessaloniki,

Thessaloniki, and

'Dept of Pneumonology, Medical School, University of Thrace Alexandroupolis, Greece.

CORRESPONDENCE

D. Bouros

Dept of Pneumonology Medical School

Democritus University of Thrace Alexandroupolis 68100 Greece

Fax: 302551076106

E-mail: bouros@med.duth.gr

Received:

August 072007

Accepted after revision:

November 262007

STATEMENT OF INTEREST

None declared. 
detection of subclinical abnormalities [15]. Furthermore, TDIderived mitral annular velocities are of proven worth in the estimation of LV filling pressures and provision of prognostic information in various cardiovascular diseases [16, 17].

On these grounds, the main aim of the present study was to determine LV and RV systolic and diastolic function in IPF patients.

\section{MATERIALS AND METHODS}

Patients (mean (range) age 66 (53-75) yrs; $n=22$ (15 male)) with IPF were studied. The diagnosis of IPF was based on the American Thoracic Society/European Respiratory Society major and minor criteria [18]. For high-resolution computed tomographic criteria, appearances compatible with fibrotic idiopathic interstitial pneumonia were required, as previously described $[19,20]$. The patients were clinically stable and ambulatory at the time of echocardiographic study and were recruited from the outpatient respiratory failure clinic of the Respiratory Failure Unit of the Aristotle University of Thessaloniki (Thessaloniki, Greece) over a period of 18 months. Throughout the study, they continued to receive the same medical treatment.

Patients with a history of significant known coronary artery disease, cardiomyopathy, valvular heart disease, arterial hypertension and symptoms/signs of RV failure were excluded from further evaluation. Furthermore, subjects showing atrial fibrillation and other rhythm abnormalities were excluded from the study.

The control group consisted of 22 age- and sex-matched control subjects without any history of pulmonary and/or cardiac disease. An important technical inclusion criterion of the study was an adequate tricuspid valve regurgitation Doppler signal, in order to assess pulmonary artery systolic pressure in all subjects enrolled in the study.

The ethics committee of the Aristotle University of Thessaloniki approved the present study. All participants gave written informed consent.

\section{Pulmonary function studies}

All patients underwent spirometry (Transfer Screen II; Jaeger, Würzburg, Germany) within 1 week of the echocardiographic study according to current guidelines [21]. Arterial blood was taken with the patient sitting and breathing room air; blood $\mathrm{pH}$ and arterial carbon dioxide and oxygen tension were measured using a commercially available blood gas analyser (ABL5; Radiometer, Copenhagen, Denmark). The functional capacity of subjects was quantitatively measured by performing the 6min walking test according to current guidelines [22].

\section{Echocardiographic study}

All patients and controls underwent a complete echocardiographic study, including two-dimensional (2D), colour-flow and spectral Doppler, as well as TDI using a GE Vingmed Vivid 7 system (GE Vingmed Ultrasound, Horten, Norway). All images were saved digitally in raw data format to magneto-optical disks for offline analysis. Brachial systolic and diastolic blood pressure measurements in the sitting position were performed using a standard mercury sphygmomanometer before each echo evaluation.
Standard 2D and colour-flow Doppler images were obtained using the parasternal long- and short-axis and apical views. Mmode traces were recorded at a speed of $50 \mathrm{~mm} \cdot \mathrm{s}^{-1}$. Three consecutive cycles were averaged for every parameter. LV diameter and wall thickness were measured from 2D targeted M-mode echocardiography according to the principal recommendations of the American Society of Echocardiography [23]. Resting LV ejection fraction was obtained using a modified Simpson's biplane method. The RV end-diastolic diameter and $\mathrm{RV}$ free-wall thickness were measured from the 2D parasternal long-axis view. RV end-systolic and end-diastolic areas were measured from the apical four-chamber view in order to calculate RV fractional area change. Pulmonary artery systolic pressure $(P$ pa,sys $)$ was estimated by calculating the maximal velocity of the tricuspid regurgitant jet and then, further using the Bernoulli equation, adding to this value an estimated right atrial pressure based on both the size of the inferior vena cava and the change in diameter of this vessel during respiration [24].

Pulsed Doppler echocardiography for the assessment of the standard diastolic filling velocities of both ventricles was performed using the apical four-chamber view. Thus the peak early diastolic filling velocity (E-wave), peak late diastolic filling velocity (A-wave), their ratio (E/A) and E-wave deceleration time (DT) were recorded. Additionally, colour M-mode was used to obtain the LV propagation velocity ( $\left.V^{\prime} \mathrm{p}\right)$,

\begin{tabular}{|c|c|c|}
\hline & IPF patients & Controls \\
\hline Subjects $\mathbf{n}$ & 22 & 22 \\
\hline Male sex n (\%) & $15(68.2)$ & $13(59.1)$ \\
\hline Age yrs & $65 \pm 9$ & $66 \pm 6$ \\
\hline $\mathrm{BMI} \mathbf{k g} \cdot \mathrm{m}^{-2}$ & $27 \pm 3$ & $28 \pm 4$ \\
\hline Ex-smokers \% & 23 & 32 \\
\hline Nonsmokers \% & 77 & 50 \\
\hline Cardiac frequency beats $\min ^{-1}$ & $77 \pm 8$ & $70 \pm 4$ \\
\hline Systolic BP mmHg & $129 \pm 7$ & $131 \pm 8$ \\
\hline Diastolic BP mmHg & $82 \pm 4$ & $84 \pm 5$ \\
\hline \multicolumn{3}{|l|}{ Lung function parameters $\%$ pred } \\
\hline TLC & $55 \pm 15$ & NA \\
\hline FVC & $61 \pm 15$ & NA \\
\hline $\mathrm{FEV}_{1}$ & $69 \pm 14$ & NA \\
\hline Residual volume & $52 \pm 16$ & NA \\
\hline$D\llcorner, C O$ & $49 \pm 22$ & NA \\
\hline \multicolumn{3}{|l|}{ Resting gas exchange $\mathrm{mmHg}$} \\
\hline $\mathrm{Pa}_{1} \mathrm{O}_{2}$ & $62 \pm 6$ & NA \\
\hline $\mathrm{Pa}_{1} \mathrm{CO}_{2}$ & $38 \pm 3$ & NA \\
\hline \multicolumn{3}{|l|}{ 6-min walking test } \\
\hline Distance m & $450 \pm 147$ & NA \\
\hline $\mathrm{O}_{2}$ desaturation \% & $6.7 \pm 2.6$ & NA \\
\hline
\end{tabular}

Data are presented as mean $\pm \mathrm{SD}$, unless otherwise indicated. IPF: idiopathic pulmonary fibrosis; BMI: body mass index; BP: blood pressure; \% pred: \% predicted; TLC: total lung capacity; FVC: forced vital capacity; FEV1: forced expiratory volume in one second; $D \mathrm{~L}, \mathrm{CO}$ : diffusing capacity of the lung for carbon monoxide; $\mathrm{Pa}, \mathrm{O}_{2}$ : arterial oxygen tension; $\mathrm{Pa}_{1} \mathrm{CO}_{2}$ : arterial carbon dioxide tension; NA: not applicable. $1 \mathrm{mmHg}=0.133 \mathrm{kPa}$. 
which serves as another index of LV diastolic function [13]. All measurements from three end-expiratory cycles were averaged at a sweep speed of $100 \mathrm{~mm} \cdot \mathrm{s}^{-1}$.

Pulsed-wave TDI was used to assess mitral and tricuspid annular velocities. Filters were set to exclude high-frequency signals, and the Nyquist limit was adjusted to a velocity range of $15-20 \mathrm{~cm} \cdot \mathrm{s}^{-1}$. Gains were minimised in order to allow for a clear tissue signal with minimum background noise. All TDI recordings were obtained during normal respiration. A 5-mm sample volume was placed at the apical four-chamber view on the lateral corner of the mitral and tricuspid annulus. The peak myocardial velocities during systole, early diastole (Em), late diastole $(\mathrm{Am})$ and their ratio $(\mathrm{Em} / \mathrm{Am})$ were recorded at a sweep speed of $100 \mathrm{~mm} \cdot \mathrm{s}^{-1}$. Velocities at the septal corner of the mitral annulus were not evaluated due to a possible interaction of the disturbed RV function. Furthermore, the ratio of $\mathrm{E}$ to $\mathrm{Em}$ (LV E/Em index) was calculated.

\section{Statistical analysis}

Data are presented as mean $\pm \mathrm{SD}$, with frequencies given as percentages. All continuous variables were normally distributed. Differences between groups were assessed using an unpaired t-test. Categorical variables were compared using a Chi-squared test or Fisher's exact test, as appropriate. Pearson's correlation coefficients were calculated for pairs of continuous variables. A p-value of $<0.05$ was considered significant and two-tailed $\mathrm{p}$-values were used throughout.

\section{RESULTS}

\section{Study population characteristics}

Of the total 32 IPF patients evaluated and characterised, 22 were finally included in the present study (table 1). Five patients were excluded due to an inadequate echocardiographic tricuspid

\begin{tabular}{|c|c|c|c|}
\hline TABLE 2 & $\begin{array}{l}\text { ntricular e } \\
\text { its with idi } \\
\text { d normal }\end{array}$ & $\begin{array}{l}\text { ardiographic } \\
\text { hic pulmona } \\
\text { bls }\end{array}$ & $\begin{array}{l}\text { ameters } \\
\text { rosis }\end{array}$ \\
\hline & Controls & IPF patients & $p$-value \\
\hline Subjects $\mathbf{n}$ & 22 & 22 & \\
\hline Area change $\%$ & $57 \pm 6$ & $42 \pm 5$ & $<0.001$ \\
\hline WT cm & $0.38 \pm 0.15$ & $0.56 \pm 0.12$ & 0.001 \\
\hline EDD cm & $2.3 \pm 0.1$ & $2.7 \pm 0.3$ & $<0.001$ \\
\hline Ppa,sys $\mathrm{mmHg}$ & $24 \pm 2$ & $47 \pm 12$ & $<0.001$ \\
\hline$E / A$ & $1.4 \pm 0.1$ & $0.7 \pm 0.07$ & $<0.001$ \\
\hline DT ms & $216 \pm 26$ & $248 \pm 42$ & 0.004 \\
\hline IVRT ms & $49 \pm 9$ & $59 \pm 15$ & $<0.001$ \\
\hline $\mathrm{Sm} \mathrm{cm} \cdot \mathbf{s}^{-1}$ & $10.4 \pm 1.0$ & $15.0 \pm 3.0$ & $<0.001$ \\
\hline $\mathrm{Em} \mathrm{cm} \cdot \mathrm{s}^{-1}$ & $11.0 \pm 2.0$ & $9.6 \pm 3.0$ & 0.08 \\
\hline$A m ~ c m \cdot s^{-1}$ & $7.1 \pm 1.9$ & $17.6 \pm 4.3$ & $<0.001$ \\
\hline $\mathrm{Em} / \mathrm{Am}$ & $1.5 \pm 0.4$ & $0.54 \pm 0.1$ & $<0.001$ \\
\hline
\end{tabular}

Data are presented as mean $\pm \mathrm{SD}$, unless otherwise stated. WT: wall thickness; EDD: end-diastolic diameter; Ppa,sys: systolic pulmonary artery pressure; E: early diastolic peak filling velocity; A: late diastolic peak filling velocity; DT: deceleration time; IVRT: isovolumic relaxation time; Sm: systolic peak myocardial velocity; Em: early diastolic peak myocardial velocity; Am: late diastolic peak myocardial velocity. $1 \mathrm{mmHg}=0.133 \mathrm{kPa}$. regurgitation Doppler signal, two due to arterial hypertension, one due to severe mitral valve regurgitation, one due to a history of coronary artery disease and one due to atrial fibrillation. The mean time from initial diagnosis of the disease was 9 months.

Patients with IPF did not differ significantly from controls in terms of body mass index, smoking habits, baseline cardiac frequency and blood pressure. Table 1 presents the lung function parameters, resting gas exchange and 6-min walking test data of patients with IPF. Amongst IPF patients at the time of the initial evaluation, five were on acetylcysteine therapy, six on azathioprine, nine on prednisone and five on intermittent oxygen therapy.

\section{RV function assessment}

It was documented that IPF patients exhibited impairment of both systolic and diastolic RV function compared with controls (table 2). They showed worse RV area change, greater RV chamber dilatation and more RV free-wall hypertrophy, all findings are indicative of a RV remodelling process secondary to increased afterload. This increased afterload was established by the higher $P$ pa,sys in patients compared with controls. None of the present patients showed a distorted (D-shaped) septum. Additionally a characteristic reversal of E/A ratio was found in IPF patients, reflecting a progressively greater contribution of late diastole to RV filling.

\begin{tabular}{|c|c|c|c|c|}
\hline \multirow[t]{2}{*}{ TABLE 3} & \multicolumn{4}{|c|}{$\begin{array}{l}\text { Left ventricular echocardiographic parameters in } \\
\text { patients with idiopathic pulmonary fibrosis (IPF) } \\
\text { and normal controls }\end{array}$} \\
\hline & & Controls & IPF patients & p-value \\
\hline \multicolumn{2}{|l|}{ Subjects n } & 22 & 22 & \\
\hline \multicolumn{2}{|l|}{ IVSd cm } & $1.1 \pm 0.2$ & $1.0 \pm 0.1$ & NS \\
\hline \multicolumn{2}{|l|}{ EDD cm } & $4.7 \pm 0.5$ & $4.8 \pm 0.7$ & NS \\
\hline \multicolumn{2}{|l|}{ EF \% } & $70 \pm 6$ & $70 \pm 5$ & NS \\
\hline \multicolumn{2}{|l|}{$E \mathrm{~m} \cdot \mathrm{s}^{-1}$} & $0.8 \pm 0.1$ & $0.6 \pm 0.1$ & $<0.001$ \\
\hline \multicolumn{2}{|l|}{$A \cdot m \cdot s^{-1}$} & $0.5 \pm 0.1$ & $0.8 \pm 0.1$ & $<0.001$ \\
\hline \multicolumn{2}{|l|}{$E / A$} & $1.5 \pm 0.1$ & $0.7 \pm 0.2$ & $<0.001$ \\
\hline \multicolumn{2}{|l|}{ DT ms } & $169 \pm 15$ & $217 \pm 16$ & $<0.001$ \\
\hline \multicolumn{5}{|c|}{ Type of diastolic dysfunction $n$} \\
\hline \multicolumn{2}{|l|}{ Normal } & $22(100)$ & & \\
\hline \multicolumn{2}{|c|}{ Impaired relaxation } & & $20(91)$ & \\
\hline \multicolumn{2}{|c|}{ Pseudonormal } & & $2(9)$ & \\
\hline \multicolumn{5}{|c|}{ Restrictive } \\
\hline \multicolumn{2}{|l|}{$V^{\prime} p \mathrm{~cm} \cdot \mathbf{s}^{-1}$} & $83 \pm 21$ & $46 \pm 13$ & $<0.001$ \\
\hline \multicolumn{2}{|l|}{$\mathrm{Sm} \mathrm{cm} \cdot \mathbf{s}^{-1}$} & $9.2 \pm 1.0$ & $8.7 \pm 3.0$ & NS \\
\hline \multicolumn{2}{|l|}{$\mathrm{Em} \mathrm{cm} \cdot \mathrm{s}^{-1}$} & $10.3 \pm 1.6$ & $5.7 \pm 1.1$ & $<0.001$ \\
\hline \multicolumn{2}{|l|}{$A m \mathrm{~cm} \cdot \mathrm{s}^{-1}$} & $5.5 \pm 0.8$ & $8.9 \pm 1.3$ & $<0.001$ \\
\hline \multicolumn{2}{|l|}{$\mathrm{Em} / \mathrm{Am}$} & $1.9 \pm 0.5$ & $0.6 \pm 0.1$ & $<0.001$ \\
\hline \multicolumn{2}{|l|}{$\mathrm{E} / \mathrm{Em}$} & $6.0 \pm 0.6$ & $10.8 \pm 3.0$ & $<0.001$ \\
\hline
\end{tabular}

Data are presented as mean \pm SD or $n(\%)$, unless otherwise stated. IVSd: interventricular septal thickness at end diastole; EDD: end-diastolic diameter EF: ejection fraction; $E$ : early diastolic peak filling velocity; A: late diastolic peak filling velocity; DT: deceleration time; $V$ 'p: propagation velocity; Sm: systolic peak myocardial velocity; Em: early diastolic peak myocardial velocity; Am: late diastolic peak myocardial velocity; Ns: nonsignificant. 


\begin{tabular}{lcc} 
TABLE 4 & $\begin{array}{l}\text { Bivariate correlations between left ventricular } \\
\text { echocardiographic parameters and pulmonary } \\
\text { artery systolic pressures }\end{array}$ \\
n & p-value \\
\hline E & -0.55 & $<0.001$ \\
A & 0.50 & 0.001 \\
E/A & -0.61 & $<0.001$ \\
DT & 0.56 & $<0.001$ \\
V'p & -0.60 & $<0.001$ \\
Sm & 0.26 & 0.08 \\
Em & -0.55 & $<0.001$ \\
Am & 0.59 & $<0.001$ \\
Em/Am & -0.64 & $<0.001$ \\
E/Em & 0.04 & 0.04 \\
\hline &
\end{tabular}

\section{LV function assessment}

A significant difference was observed between the two groups as regards E/A ratio, DT of the E-wave and $V^{\prime}$, reflecting early diastolic filling impairment in this specific population (table 3). The two groups did not differ significantly in terms of LV dimensions and systolic function.

Mitral annular TDI analysis confirmed the presence of LV diastolic dysfunction in IPF patients, with lower LV Em and Em/ Am ratios than controls. Moreover, patients with IPF showed higher E/Em ratios than controls. Conversely, peak mitral annular systolic velocity was similar between the two groups.

\section{Correlations}

Bivariate correlation analysis revealed significant relations between indices of LV diastolic function and $P$ pa,sys (table 4). The negative correlations of LV E/A ratio $(r=-0.61 ; \mathrm{p}<0.001)$, $\mathrm{Em} / \mathrm{Am}$ ratio $(\mathrm{r}=-0.64 ; \mathrm{p}<0.001)$ and $V^{\prime} \mathrm{p}(\mathrm{r}=-0.60 ; \mathrm{p}<0.001)$ with $P$ pa,sys are of note (fig. 1 ).

\section{DISCUSSION}

In the present study, it was demonstrated that patients with clinically stable IPF exhibit not only RV diastolic and systolic dysfunction but also impaired LV diastolic filling. Conversely, LV systolic function seems to be preserved. The present authors believe that the current findings have important implications for the management, and possibly the prognosis, of patients with IPF.

To the best of the present authors' knowledge, there are no reports in the literature regarding any association between IPF and LV diastolic function. The present findings from both standard Doppler (E/A ratio) and less load-dependent techniques [13, 15], such as colour M-mode ( $\left.V^{\prime} \mathrm{p}\right)$ and TDI (Em, E/Em and Em/Am), suggest that LV diastolic function, particularly early relaxation, is impaired in these patients. Similar disturbances in LV diastolic filling were also reported in previous studies evaluating LV diastolic performance in diseases that mainly affect RV function [5-7, 11, 12].
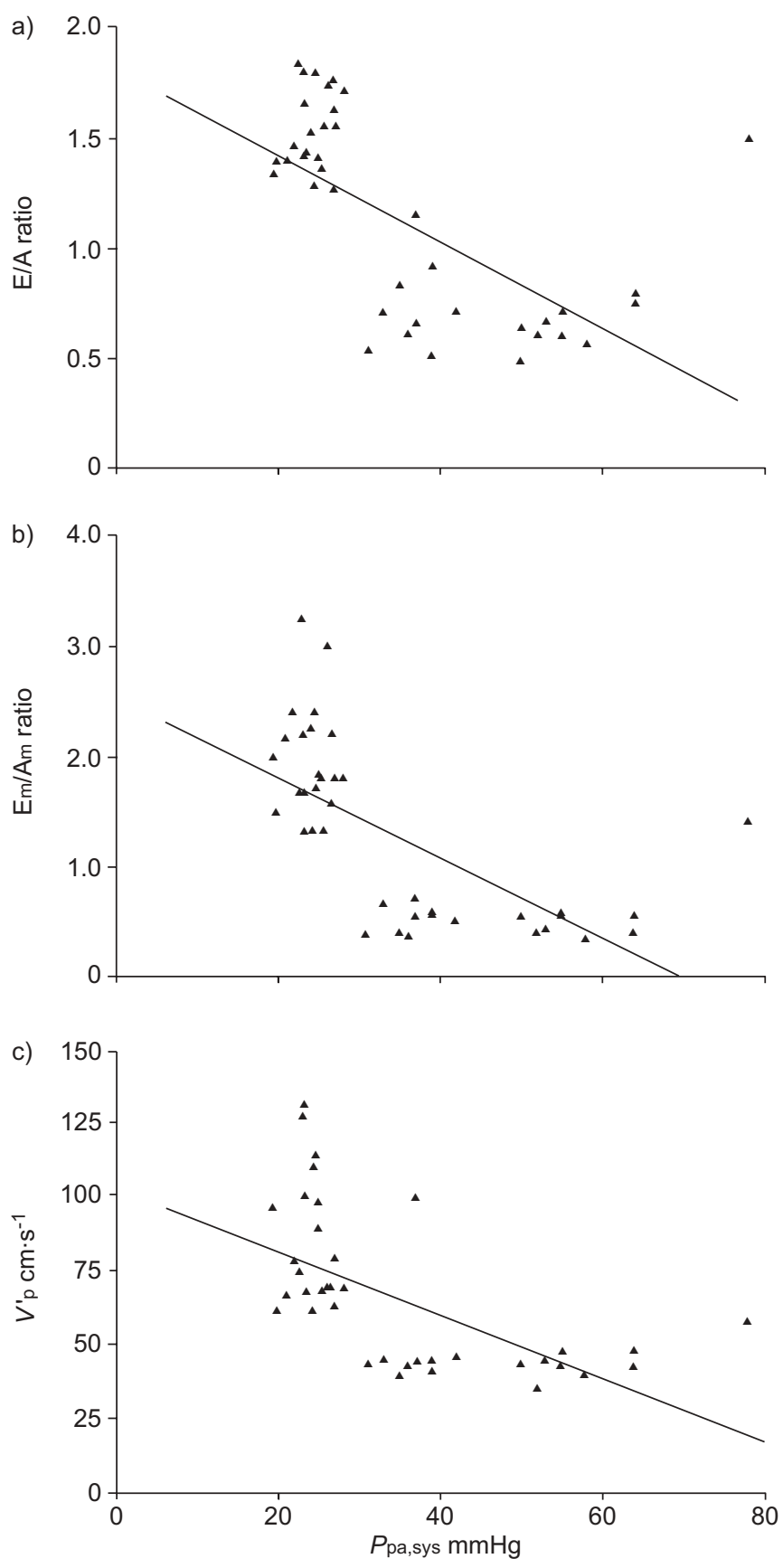

FIGURE 1. Scatter diagrams showing the correlation between systolic pulmonary artery pressure (Ppa,sys) and left ventricular: a) early diastolic peak filling velocity $(E) / l a t e$ diastolic peak filling velocity $(A)$ ratio $(r=-0.61 ; p<0.001)$; b) early diastolic peak myocardial velocity $(\mathrm{Em}) / \mathrm{late}$ diastolic peak myocardial velocity $(\mathrm{Am})$ ratio $(r=-0.64 ; \mathrm{p}<0.001)$; and $\mathrm{c})$ propagation velocity $(\mathrm{V} p ; r=-0.60$ $\mathrm{p}<0.001) .1 \mathrm{mmHg}=0.133 \mathrm{kPa}$.

Conversely, LV systolic function, as measured by both standard 2D and TDI echocardiography, was preserved in the present IPF patients, another finding that is in keeping with previous reports evaluating LV systolic function in clinical entities that affect RV performance [4-12].

It is well known that diastolic dysfunction, particularly in the early phase of diastole, is the most common type of LV 
dysfunction seen in patients with chronic PH [25]. This impairment of early relaxation could be explained by different mechanisms. One of these is the distortion of the interventricular septum towards the LV as the RV adapts to pressure or volume overload and increases in size and mass [26]. This is less likely in the present study population since $\mathrm{PH}$ was only mild to moderate. Secondly, since the RV and LV operate as a syncytium, the diastolic function of one ventricle may influence that of the other, and this phenomenon is well recognised as ventricular interdependence [26, 27]. The present finding of significant association between $P$ pa,sys and LV diastolic function indices underlines the presence of ventricular interdependence in patients with IPF. Finally, compensatory neurohormonal activation seems to occur while RV function declines, and this may influence loading conditions in both ventricles. In more detail, it has been shown, in experimental settings, that peptides with positive inotropic effect, such as angiotensin II, endothelin 1, atrial natriuretic peptide and noradrenalin, are produced. These substances may affect the remodelling process of both ventricles, by changing their loading conditions, inducing collagen synthesis and inhibiting collagen degradation [28, 29].

Patients in the early stages of IPF may demonstrate normal or only slightly elevated Ppa,sys [1-3], but, with the progression of the disease, pulmonary pressure increases dramatically within months [2]. Therefore, it is important to determine, in the early stages of the disease, whether these patients suffer from LV diastolic dysfunction, and, further, to investigate, whether or not there is any association with the severity of $\mathrm{PH}$ and consequently with IPF disease progression. The present finding of significant associations between LV diastolic function indices and $P$ pa,sys further supports this hypothesis. If this is the case, echocardiographic parameters of LV diastolic dysfunction could be routinely used for risk stratification and therapeutic monitoring and guidance in such cohorts. Moreover, young patients who have successfully undergone lung transplantation with long-term survival from improved allograft preservation may eventually present with symptomatic left heart failure due to progressive LV diastolic dysfunction. Thus study of LV function in IPF patients after transplantation is warranted.

\section{Limitations}

The main limitation of the present study is that the evaluation of $\mathrm{PH}$ was performed using Doppler echocardiography and not right heart catheterisation, which is the gold standard method. Moreover, mitral annular TDI recordings could be influenced by overall heart motion and the contraction of adjacent myocardial segments, rendering this modality less sensitive than strain rate imaging [30].

In conclusion, patients with idiopathic pulmonary fibrosis exhibit predominantly type I left ventricular diastolic dysfunction (impaired early relaxation) in addition to the expected impairment of right ventricular systolic and diastolic function. Whether or not left ventricular diastolic abnormalities have prognostic implications for the clinical course of patients with idiopathic pulmonary fibrosis remains to be investigated. The present authors believe that serial echocardiographic measurements, particularly using tissue Doppler imaging, are warranted in this population in order to follow the progression of cardiac dysfunction.

\section{REFERENCES}

1 Noble PW, Homer RJ. Idiopathic pulmonary fibrosis: new insights into pathogenesis. Clin Chest Med 2004; 25: 749-758.

2 King TE Jr, Tooze JA, Schwarz MI, Brown KR, Cherniack RM. Predicting survival in idiopathic pulmonary fibrosis: scoring system and survival model. Am J Respir Crit Care Med 2001; 164: 1171-1181.

3 Bouros D, Antoniou KM. Current and future therapeutic approaches in idiopathic pulmonary fibrosis. Eur Respir J 2005; 26: 693-702.

4 Lettieri CJ, Nathan SD, Barnett SD, Ahmad S, Shorr AF. Prevelance and outcomes of pulmonary arterial hypertension in advanced idiopathic pulmonary fibrosis. Chest 2006; 129: 746-752.

5 Bossone E, Duong-Wagner TH, Paciocco G, et al. Echocardiographic features of primary pulmonary hypertension. J Am Soc Echocardiogr 1999; 12: 655-662.

6 Yilmaz R, Gencer M, Ceylan E, Demirbag R. Impact of chronic obstructive pulmonary disease with pulmonary hypertension on both left ventricular systolic and diastolic performance. J Am Soc Echocardiogr 2005; 18: 873-881.

7 Menzel T, Wagner S, Kramm T, et al. Pathophysiology of impaired right and left ventricular function in chronic embolic pulmonary hypertension: changes after pulmonary thromboendarterectomy. Chest 2000; 118: 897-903.

8 Lindqvist P, Caidahl K, Neuman-Andersen G, et al. Disturbed right ventricular diastolic function in patients with systemic sclerosis: a Doppler tissue imaging study. Chest 2005; 128: 755-763.

9 Gin PL, Wang WC, Yang SH, Hsiao SH, Tseng JC. Right heart function in systemic lupus erythematosus: insights from myocardial Doppler tissue imaging. I Am Soc Echocardiogr 2006; 19: 441-449.

10 Ionescu AA, Ionescu AA, Payne N, Obieta-Fresnedo I, Fraser AG, Shale DJ. Subclinical right ventricular dysfunction in cystic fibrosis. A study using tissue Doppler echocardiography. Am J Respir Crit Care Med 2001; 163: 1212-1218.

11 D'Andrea A, Stisi S, Bellissimo S, et al. Early impairment of myocardial function in systemic sclerosis: non-invasive assessment by Doppler myocardial and strain rate imaging. Eur J Echocardiogr 2005; 6: 407-418.

12 Mahmud E, Raisinghani A, Hassankhani A, et al. Correlation of left ventricular diastolic filling characteristics with right ventricular overload and pulmonary artery pressure in chronic thromboembolic pulmonary hypertension. J Am Coll Cardiol 2002; 40: 318-324.

13 Garcia MJ, Thomas JD, Klein AL. New Doppler echocardiographic applications for the study of diastolic function. J Am Coll Cardiol 1998; 32: 865-875.

14 Nagueh SF, Middleton KJ, Kopelen HA, Zoghbi WA, Quinones MA. Doppler tissue imaging: a non-invasive technique for evaluation of left ventricular relaxation and estimation of filling pressures. J Am Coll Cardiol 1997; 30: 1527-1533.

15 Sutherland GR, Lange A, Palka P, Grubb N, Fleming A, McDicken WN. Does Doppler myocardial imaging give new insights or simply old information revisited. Heart 1996; 76: 197-199. 
16 Hillis GS, Møller JE, Pellikka PA, et al. Noninvasive estimation of left ventricular filling pressure by $E / e^{\prime}$ is a powerful predictor of survival after acute myocardial infarction. J Am Coll Cardiol 2004; 43: 360-367.

17 Wang M, Yip GW, Wang AY, et al. Peak early diastolic mitral annulus velocity by tissue Doppler imaging adds independent and incremental prognostic value. J Am Coll Cardiol 2003; 41: 820-826.

18 American Thoracic Society, European Respiratory Society. American Thoracic Society/European Respiratory Society international multidisciplinary consensus classification of the idiopathic interstitial pneumonias. Am J Respir Crit Care Med 2002; 165: 277-304.

19 Wells AU, Rubens MB, du Bois RM, Hansell DM. Serial CT in fibrosing alveolitis: prognostic significance of the initial pattern. AJR Am J Roentgenol 1993; 161: 1159-1165.

20 Wells AU, Hansell DM, Rubens B, Cullinan P, Black CM, $\mathrm{du}$ Bois RM. The predictive value of appearances on thin section computed tomography in fibrosing alveolitis. Am Rev Respir Dis 1993; 148: 1076-1082.

21 Standarization of spirometry, 1994 update. American Thoracic Society. Am J Respir Crit Care Med 1995; 152: 1107-1136.

22 ATS Committee on Proficiency Standards for Clinical Pulmonary Function Laboratories, ATS statement: guidelines for the six-minute walk test. Am J Respir Crit Care Med 2002; 166: 111-117.

23 Schille NB. Two-dimensional echocardiographic determination of left ventricular volume, systolic function and mass.
Summary and discussion of the 1989 recommendations of the American Society of Echocardiography. Circulation 1991; 83: Suppl. 3, 1280-1287.

24 Schiller NB. Pulmonary artery pressure estimation by Doppler and two-dimensional echocardiography. Cardiol Clin 1990; 8: 277-287.

25 Louie EK, Lin SS, Reynertson SI, Brundage BH, Levitsky S, Rich S. Pressure and volume loading of the right ventricle have opposite effects on left ventricular ejection fraction. Circulation 1995; 92: 819-824.

26 Morris-Thurgood J, Frenneaux M. Diastolic ventricular interaction and ventricular diastolic filling. Heart Fail Rev 2000; 5: 307-323.

27 Lazar JM, Flores AR, Grandis DJ, Orie JE, Schulman DS. Effects of chronic right ventricular pressure overload on left ventricular diastolic function. Am J Cardiol 1993; 72: 1179-1182.

28 Lamberts RR, Vaessen RJ, Westerhof N, Stienen GJM. Right ventricular hypertrophy causes impairment of left ventricular diastolic function in the rat. Basic Res Cardiol 2007; 102: 19-27.

29 Brunner F. Cardiac endothelin in right-heart hypertrophy due to monocrotaline-induced pulmonary hypertension in rat. Cardiovasc Res 1999; 44: 197-206.

30 Sutherland GR, Di Salvo G, Claus P, D'hooge J, Bijnens B. Strain and strain rate imaging: a new clinical approach to quantifying regional myocardial function. J Am Soc Echocardiogr 2004; 17: 788-802. 